\title{
EFFECTS OF COMPETITION AND PREDATION IN A THREE SPECIES MODEL
}

\author{
JANUSZ SZWABIŃSKI, ANDRZEJ PĘKALSKI and KAMIL TROJAN \\ Institute of Theoretical Physics, University of Wrockaw \\ M. Borna 9, 50-204 Wrockaw, Poland \\ E-mail: szwabin@ift.uni.wroc.pl,apekal@ift.uni.wroc.pl,ktrojan@ift.uni.wroc.pl
}

\begin{abstract}
A model which consists of a predator and two prey species is presented. The prey compete for the same limited resource (food). The predator preys on both prey species but with different severity. We show that the coexistence of all three species is possible in a mean-field approach, whereas from Monte Carlo simulation it follows that the stochastic fluctuations drive one of the prey populations into extinction.
\end{abstract}

1. Introduction. One of the most important problems in ecology is to investigate the question of coexistence of species. Starting with the pioneering works of Lotka [1] and Volterra [12] there are numerous papers by either biologists, mathematicians or physicists studying the dynamics of predator-prey systems or systems with competition (see e.g. $[7,1,2,5,10,8])$. There are also some studies on three species food chains (e.g. [4]). However, to the best of our knowledge an exhaustive analysis of three species models with both predation and competition interactions is still missing. Understanding the interplay between these two mechanisms is of great practical interest, since it could suggest methods for manipulating populations in biological control.

In this paper we present a model of three populations living in the same ecosystem - two of them are prey and one is a predator population. The prey populations differ in one aspect only - one is preferred by the predators as food. Since it is known [3] that sometimes in biological models results obtained via mean-field type approach are even qualitatively different from those coming from agent-based simulations models, we use here therefore two methods - mean-field leading to differential equations and simulations based on treating each individual independently.

2000 Mathematics Subject Classification: Primary 92D25.

Key words and phrases: predator-prey model, Monte Carlo simulation.

The paper is in final form and no version of it will be published elsewhere. 
2. Mean-field type model. The ecosystem that we model consists of a predator $(P R)$ and two prey species ( $P 1$ and $P 2)$. The prey species compete for the same limited resource (living space) but have unlimited access to food. Both prey species are food for the predators, but with different preference. The equations defining the dynamics of the model we study are:

$$
\begin{aligned}
& \frac{\mathrm{d} N_{1}}{\mathrm{~d} t}=r N_{1}\left[1-\frac{N_{1}}{K}-b \frac{N_{2}}{K}-c_{1} N_{3}\right], \\
& \frac{\mathrm{d} N_{2}}{\mathrm{~d} t}=r N_{2}\left[1-\frac{N_{2}}{K}-b \frac{N_{1}}{K}-c_{2} N_{3}\right], \\
& \frac{\mathrm{d} N_{3}}{\mathrm{~d} t}=r_{3} N_{3}\left[c_{1} N_{1}+c_{2} N_{2}-d\right],
\end{aligned}
$$

where $N_{1}, N_{2}$ and $N_{3}$ are the numbers of elements of the $P 1, P 2$ and $P R$ populations, respectively. The parameters $r$ and $r_{3}$ are the natural birth rates of the species, $d$ is the mortality rate of the predators and $K$ the carrying capacity. The constant $b$ represents effects of competition between $P 1$ and $P \mathscr{2}$. Predation rates are given by $c_{1}$ and $c_{2}$. Substituting the rescalings $x=N_{1} / K, y=N_{2} / K, z=N_{3} / K$, and $\tau=r t$ we get

$$
\begin{aligned}
& \frac{\mathrm{d} x}{\mathrm{~d} \tau}=x[1-x-b y-\alpha z], \\
& \frac{\mathrm{d} y}{\mathrm{~d} \tau}=y[1-y-b x-\beta z], \\
& \frac{\mathrm{d} z}{\mathrm{~d} \tau}=\gamma z[\alpha x+\beta y-d],
\end{aligned}
$$

where $\alpha=c_{1} K, \beta=c_{2} K$ and $\gamma=r_{3} / r$. The nullclines analysis of Eq. (2) leads to seven fixed points, the first of which is the trivial empty state $P_{1}=(0,0,0)$. The next two points are states with only one prey species present: $P_{2}=(1,0,0)$ and $P_{3}=(0,1,0)$. In addition, there are three points with one species absent: $P_{4}=\left(\frac{1}{b+1}, \frac{1}{b+1}, 0\right), P_{5}=\left(\frac{d}{\alpha}, 0,-\frac{d-\alpha}{\alpha^{2}}\right)$ and $P_{6}=\left(0, \frac{d}{\beta},-\frac{d-\beta}{\beta^{2}}\right)$. And finally, there is exactly one steady state $P_{7}$, where all three species exist:

$$
\left(-\frac{(b \beta-\alpha) d+\beta(\alpha-\beta)}{\beta^{2}-2 \alpha \beta b+\alpha^{2}}, \frac{(\beta-\alpha b) d+\alpha(\alpha-\beta)}{\beta^{2}-2 \alpha \beta b+\alpha^{2}}, \frac{\left(b^{2}-1\right) d+(1-b)(\alpha+\beta)}{\beta^{2}-2 \alpha \beta b+\alpha^{2}}\right) .
$$

The stability of all equilibria, as well as the dynamics of the system, is of course dependent on the values of the model parameters. To focus our attention we will consider the special case

$$
\gamma=0.50, \quad b=0.20, \quad d=0.90, \quad \alpha=25
$$

and concentrate only on how the dynamics changes if the parameter $\beta$ representing predation effects on the $P \mathscr{2}$ species varies. The values (4) are chosen to roughly match the oscillation period obtained from Monte Carlo simulation (discussed briefly in the next section). In this case the points $P_{1}, P_{2}, P_{3}$ and $P_{4}$ are unstable, no matter what the value of $\beta$ is. From the local stability analysis [11] it follows that the point $P_{5}$ is stable for $\beta>\beta_{1}=25.7469$. Similarly, we can show that the equilibrium $P_{6}$ is stable if the condition $\beta<\beta_{2}=24.2523$ is satisfied. Furthermore, note that $x_{7}\left(\beta_{1}\right)=0$ and $y_{7}\left(\beta_{2}\right)=0$. Thus the interior point $P_{7}$ joins the fixed point $P_{6}$ or $P_{5}$ exactly at the transition value $\beta_{1}$ 
or $\beta_{2}$. It can be deduced from the above discussion that the coexistence is possible only in a narrow range $\beta \in(24.2523,25.7469)$ around $\alpha=25$. Indeed, one can check that for these values of $\beta$ the interior point is a stable spiral. Outside that range the prey favored by the predators as food will be eliminated. In Fig. 1, an example evolution of the system in the coexistence case is shown.

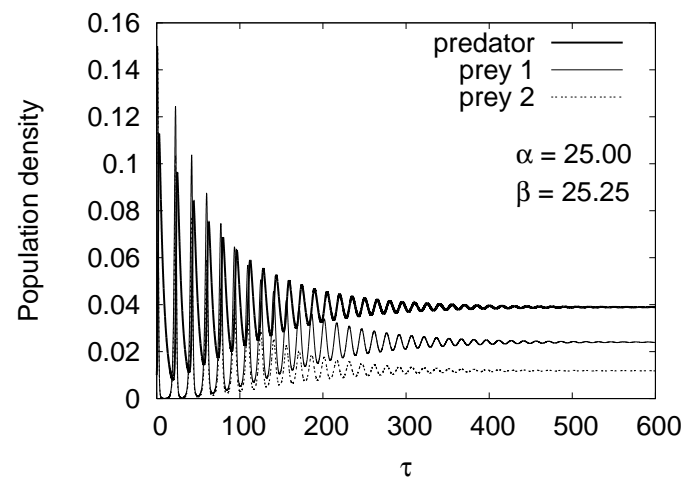

Fig. 1. Time evolution of the system in the coexistence case $\beta=25.25$. The interior point $P_{7}$ is a stable spiral.

3. Agent-based model. Let us switch now to the Monte Carlo simulation of the investigated system. Our species live now on a square $L \times L$ lattice. They move at random and interact with each other following some rules. A detailed description of these rules may be found elsewhere [11], but we should mention some of them. (i) Double occupancy of sites by prey is not allowed. In this way the competition for living space is realized. (ii) Cross-breeding between two types of prey is prohibited. (iii) Predators reproduce if their food reserves are large enough. (iv) Animals reproduce in a quasi-sexual way, i.e. there is no sexual difference among the animals of the same type. However, two of them must meet in the neighboring sites in order to breed. (v) Prey is killed either with probability 1 , or with probability $1-p$, where the parameter $p$ corresponds to the difference $|\alpha-\beta|$ in the mean-field aproach.

The model has the following parameters: (i) linear size $L$ of the system, (ii) initial concentrations of the animals, (iii) birth rate $m$ for prey, (iv) maximum food reserves $f$ or alternatively, number of MCS a predator may live without catching prey, (v) minimum food reserves $f m$ necessary for breeding, (vi) food reserves $f b$ received by a baby predator and (vii) preference $p$ in killing by predators of prey 1 over prey 2 . It would be of course quite difficult, if not impossible, to investigate our model in the space of all those parameters. We have therefore decided to fix most of them and vary only $L$ and $p$. The values taken for the remaining parameters are: initial concentrations $c(P 1)=c(P 2)=0.2, c(P R)=0.1, m=6, f=8, f m=6, f b=4$.

Our previous results show the existence of a narrow range of values of the $p$ parameter, for which the coexistence of the three species is possible. To see if this is also the case in MC simulations, we let the system evolve for a given time and different values 


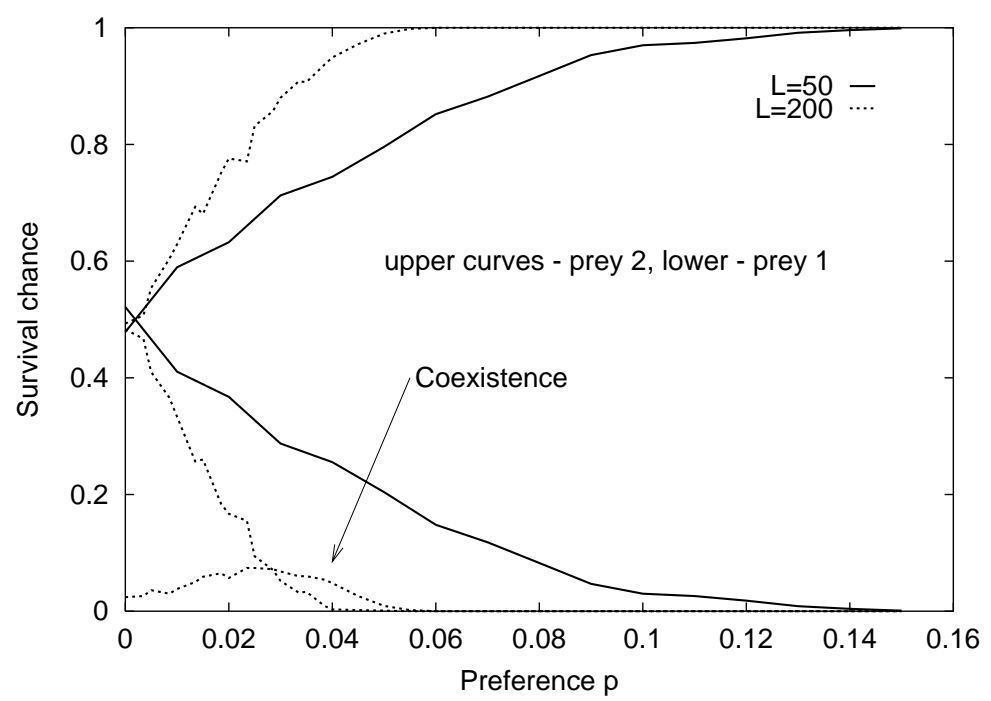

Fig. 2. Survival chance for the prey populations (at 6000 MCS)

of $p$ and recorded the final densities of the species. The results (averaged over 2000 runs for $L=50$ and 1000 runs for $L=200$ ) after 6000 MCS are shown in Fig. 2. They are presented as a survival chance, i.e. the number of cases in which a given population was still present at the end of simulations. The difference between dynamics on larger $(L=$ $200)$ and small $(L=50)$ lattices should be noticed. For a relatively large value of $p$, say, $p=0.05$, the chances of survival for $P 1$ in large lattices are nearly null. However on a small lattice they have a good chance to live on. The difference is due to stochastic fluctuations which play much more important role in small systems. This susceptibility of small populations to stochastic changes is well known in biology (see e.g. [9]). The role of the preference factor is, to some extent, specially in small systems, masked by stochastic effects in spatial arrangements of the population which may save or drive the population to extinction. The chance of coexistence is larger for bigger systems. For $L=50$ we have never, regardless of the value of $p$, found a coexistence of the three species at the end of a simulation. For a larger system we have found a rather curious dependence of the coexistence chance on the value of $p$, since it initially increases with $p$.

Only when there are no predators, the two types of prey coexist forever and have the same concentration. In other cases there is a narrow range of the values of the preference parameter $p$, for which coexistence occurs, even for very long simulation times, however not indefinitely [11]. This kind of behavior differs from the mean-field results, but is quite similar to situations encountered in nature, where competition between two populations generally drives one of them to extinction if the living conditions are hard, but has small effect if the conditions are easy. This statement has never been proved in biology and is known as the Gause principle [13]. In our model difficult conditions are realized via the presence of predators. 


\section{References}

[1] A. Arditi and L. R. Ginzburg, J. Theor. Biol. 139 (1989), 311.

[2] A. A. Barryman, Ecology 73 (1992), 1530.

[3] R. Durrett and S. Levin, Theor. Popul. Biol. 46 (1994), 363.

[4] A. Klebanoff and A. Hastings, J. Math. Biol. 32 (1994), 427

[5] A. Lipowski, Phys. Rev. E 60 (1999), 5179.

[6] A. J. Lotka, Proc. Natl. Acad. Sci. USA 6 (1920), 410.

[7] J. Maynard Smith and M. Slatkin, Ecology 54 (1972),384

[8] A. Pękalski, Computing in Science and Engineering 6 (2004), 62.

[9] M. Shaffer, BioScience 31 (1981), 131.

[10] D. Stauffer, A. Kunwar and D. Chowdhury, Physica A 352 (2005), 202.

[11] J. Szwabiński, A. Pękalski and K. Trojan, Int. J. Mod. Phys. C 17 (2006), 1629.

[12] V. Volterra, Leçons sur la Théorie Mathématique de la Lutte pour la Vie, Gauthier-Villars, Paris, 1931.

[13] M. Wiliamson, The Analysis of Biological Populations, E. Arnold, London, 1972. 
